\title{
Nivel de satisfacción y compromiso en trabajadores remunerados versus voluntariado y motivación en el mismo
}

\author{
Eduvigis Carmona Muñoz. Universidad de Granada \\ Iñaki Estellés Frade. Universidad de Granada \\ Guillermo Díaz Paredes. Universidad de Granada \\ Rubén Aguilera García. Universidad de Granada
}

\author{
Recepción: 26.02.2018 | Aceptado: 5.03.2018 \\ Correspondencia a través de ORCID: Eduvigis Carmona Muñoz iD 0000-0002-0924-4736 \\ Citar: Carmona, E., Estellés, I., Díaz, G. y Aguilera, R. (2018). Nivel de satisfacción y \\ compromiso en trabajadores remunerados versus voluntariado y motivación en el mismo. \\ ReiDoCrea, 7, 85-91.
}

Resumen: La satisfacción y el compromiso en el campo de la Psicología del Trabajo nos ha permitido conocer y mejorar las relaciones que se establecen entre los individuos y su lugar de trabajo oficio $u$ organización de la que sean partícipes. El objetivo general de esta investigación es determinar si el grado de satisfacción y compromiso varía dependiendo del tipo de actividad laboral que se desempeñe y de si estos parámetros son mayores en los voluntarios que en los trabajadores remunerados. Para ello se usaron los cuestionarios Q12 y S21/26. También nos interesa conocer qué tipo de motivación predomina en los trabajadores voluntarios por lo que se utilizó el Cuestionario de Motivación para el Voluntariado. Parece que la motivación intrínseca del grupo de los voluntarios está estrechamente relacionada con una mayor satisfacción y compromiso laboral que cuando se trata de reforzadores motivacionales de carácter extrínseco, que son los que suelen experimentar los voluntarios. Las diferencias significativas encontradas en las dos variables analizadas entre los dos grupos concuerdan con nuestra hipótesis inicial. Los voluntarios mostraban mayor compromiso y satisfacción con la labor que ostentaban que los trabajadores remunerados, observándose además entre los voluntarios una alta motivación intrínseca por la actividad que ejercían.

Palabras clave: Satisfacción | Trabajador/a

Level of Satisfaction and Commitment in Paid Workers Versus Volunteer Workers and Motivation in Volunteers

\begin{abstract}
Satisfaction and commitment in the field of work psychology have allowed us to determine and improve the relationships established between individuals and their workplace, or the profession or organization they participate in. The general objective of this research study is to determine if the degree of satisfaction and commitment varies depending on the types of work performed and if these parameters are higher in volunteers than in paid workers. To do this, questionnaires Q12 and S21 / 26 were used. We are also interested in knowing what type of motivation predominates in volunteer workers, for which the Motivation Questionnaire for Volunteering was used. It seems that the intrinsic motivation of the group of volunteers is closely related to greater satisfaction and work commitment than when it comes to motivational reinforcers of an extrinsic nature, which are what volunteers usually experience. The significant differences found in the two variables analyzed between the two groups are consistent with our initial hypothesis. Volunteers showed greater commitment and satisfaction with the work than paid workers, also observing among volunteers a high intrinsic motivation for the activity they carried out.
\end{abstract}

Keywords: Satisfaction | Workers

\section{Introducción}

Con la entrada del nuevo siglo y el cambio en la estructura de numerosas organizaciones, el significado del empleo ha sufrido cuantiosas distorsiones que han conformado un área de estudio concreta, de forma que las relaciones laborales constituyen uno de los temas más estudiados hoy en día. Cada vez más, el trabajo tiene más importancia en nuestras vidas y, cómo no, el papel que desempeñan los trabajadores dentro de él. No resulta sorprendente que surjan diversos intentos para 
entender qué variables psicológicas y sociales influyen favorablemente en los trabajadores (Mañas, Salvador, Boada, González y Agulló, 2007). Nos centraremos en estudiar la satisfacción, el compromiso y la motivación en el ambiente laboral, tanto remunerado como voluntario.

En primer lugar, se hablará de la satisfacción laboral, siendo éste un concepto largamente estudiado y discutido desde la Psicología de la Organización. Sin embargo, en la actualidad, sigue siendo un concepto controvertido sobre el cual existen tantas definiciones como autores se han interesado por él. Dentro del debate sobre la satisfacción laboral encontramos dos posturas mayoritarias, nos centraremos en la postura predominante y más usada a día de hoy para aproximarse al estudio de la satisfacción laboral. Autores como Brief y Weiss (2001) defienden que es una combinación de emoción y cognición, un estado interno que se manifiesta de una de estas dos formas; pensamientos y/o emociones. Más recientemente, Robbins (2004) la define como una actitud hacia al trabajo, esto es, un conjunto de respuestas cognitivas y afectivas hacia la situación laboral. Estas definiciones no sólo tienen en cuenta la respuesta afectiva hacia el trabajo, sino que, además, entiende que los pensamientos - la evaluación del trabajador hacia su trabajo cumplen un papel crucial en el comportamiento y la disposición de la persona para con su tarea.

En cuanto al compromiso laboral, la evidencia empírica indica que la estabilidad del servicio se consigue si las instituciones logran comprometer a sus empleados. Desde esta perspectiva, el compromiso organizacional permite estabilidad laboral, esto tiene un fuerte impacto en prestaciones, jubilación y beneficios sociales garantizando cubrir necesidades tanto materiales como psicológicas (reconocimiento, desarrollo de habilidades, socialización, estatus) beneficiando a las familias y la economía del país (Betanzos y Paz, 2007). Dentro de un marco teórico, se podría definir como la identificación psicológica de una persona con la organización en la que trabaja (Arciniega, 2002). Este compromiso es visto como una respuesta emocional ante una evaluación positiva dentro del contexto laboral por parte del trabajador. Además, han sido las aportaciones de Meyer y Allen (1984) las que han provocado un mayor impacto, los cuales diferencian tres tipos de compromiso, que difieren en sus causas y consecuencias. Conjugando los tres tipos presentados, se obtendría la siguiente idea: Los trabajadores se comprometen con una organización, permaneciendo involucrados porque así lo desean y han establecido un vínculo emocional con su centro laboral (afectivo), porque lo necesitan y se sienten obligados de permanecer en la institución (continuo) y porque sienten la obligación moral de hacerlo (normativo) (Gallardo y Triado, 2007).

Pasando ahora a la motivación, se debería de reconocer cuáles son las razones que mueven a un colectivo como es el voluntariado. El estudio de las motivaciones en este grupo podría tener un gran impacto para la gestión de los programas enfocados hacia su difusión y mantenimiento. Saber cuáles son las razones que mueven a las personas a realizar una tarea sin una remuneración económica, nos permite formar estrategias de captación y mantenimiento de éstas. Una de las variables que creemos indispensables para el estudio de las dinámicas que determinan la buena disposición en el ambiente de trabajo, es el compromiso afectivo. Este tipo de compromiso podría estar relacionado con la motivación intrínseca, el trabajador realiza una actividad por el simple placer de realizarla sin recibir algún incentivo externo, sintiendo una sensación de éxito y un fuerte deseo personal. A su vez, el compromiso continuo podría vincularse más con la motivación extrínseca, porque lo que atrae al trabajador no es la actividad que realiza en sí, sino lo que recibe a cambio por la actividad realizada, el incentivo (McDonald y Makin, 2000). 
Las motivaciones suponen un alto grado de implicación y la idea que subyace en esta estrategia es que la satisfacción de las motivaciones es la clave para la permanencia (Chacón y Vecina, 2002, Chacón, Vecina y Dávila, 2007; Clary, Snyder y Ridge, 1992; Clary, Snyder, Ridge, Miene y Haugen, 1994; Dávila y Chacón 2003; Vecina, Chacón, y Sueiro, 2009). Un cuestionario que ha tenido un gran impacto para el estudio de las motivaciones en el voluntariado fue el Volunteer Functions Inventory (VFI) propuesto por Clary et. al. (1998). Este cuestionario contiene 6 emociones distintas: Valores (interés humanitario por ayudar a otros), Conocimiento (gusto por aprender sobre temas diversos), Ajuste Social (necesidad de adaptarse a lo que personas cercanas y relevantes valoran), Mejora del Currículum, Mejora de la Estima (interés en el disfrute de sentimientos positivos) y Defensa del Yo (necesidad de protegerse y escapar de sentimientos negativos como, por ejemplo, la soledad) (Chacón, Pérez, Flores y Vecina, 2010).

La Teoría del Aprendizaje Social (Bandura, 1977) exhibe que nuestro comportamiento altruista depende en gran medida de la experiencia vivida, de manera que, si hemos experimentado consecuencias negativas o beneficios después de haber ayudado a los demás en el pasado, nuestra actitud generosa en el presente se ve modulada. En el momento en que los sujetos interiorizan los beneficios de ayudar a los demás, ya no hace falta esa motivación extrínseca. Lerner (1980) considera que nuestra actitud de ayuda hacia el resto está influida también por la situación de la otra persona, de manera que las desigualdades o pobreza nos afectan mucho, convirtiéndose aquellas personas víctimas de esas situaciones en un factor precipitante de la actitud humanitaria. La mayoría de estudios relacionados con la motivación en el voluntariado nos muestran que ésta se compone de una combinación entre motivaciones orientadas a los demás o altruismo y motivaciones relacionadas con el beneficio propio (Valor-Segura, 2011). De hecho, la Teoría Funcional aplicada a este tema propone que nunca el comportamiento del voluntario va a depender de un solo factor, siendo los principales causantes: 1. los valores del individuo; 2. la necesidad de adaptación a un grupo; 3. la necesidad de adquirir experiencia y cierto bagaje cultural; 4. la autoprotección ante ansiedad, estrés...(Chacón et al., 2007; Clary y Snyder, 1991, 1999; López, Cábanas y Chacón, 1997; Omoto y Snyder, 1999).

\section{Objetivos o hipótesis}

El objetivo en este estudio será medir los constructos presentados con anterioridad y ver cómo correlacionan tanto con el trabajo remunerado como con el voluntariado. Así, esperamos que el grupo del voluntariado tenga mayor satisfacción y compromiso con el puesto que desempeñan que los trabajadores remunerados. Intentaremos identificar los factores motivacionales predominantes que mueven a este colectivo.

\section{Métodos}

\section{Participantes}

Una muestra heterogénea compuesta por 40 personas, 20 de ellas serán trabajadores remunerados de diferentes entidades y otros 20 voluntarios también de distintas asociaciones.

\section{Instrumentos}

1. Cuestionario de Satisfacción Laboral S21/26 (Meliá y Peiró, 1998): Se trata de un cuestionario encargado de medir la satisfacción de los trabajadores. Se divide en dos secciones: la primera consta de 26 ítems con respuesta dicotómica (V/F). Por 
otro lado, la segunda parte pregunta por los datos descriptivos de los sujetos. En nuestra investigación, al ser un cuestionario completamente anónimo hemos utilizado únicamente la parte de verdadero y falso. Además, ella nos permite medir: satisfacción en la supervisión y participación en la organización, satisfacción con la remuneración y las prestaciones, satisfacción intrínseca, satisfacción con el ambiente físico, satisfacción con la cantidad de producción y satisfacción relacionada con la calidad.

2. Cuestionario de Compromiso Q12 (Gallup, 2011): Se trata de un cuestionario que mide el grado de compromiso que sienten los trabajadores en sus puestos de trabajo. Consta de 26 ítems, con una escala Likert de 5 niveles, donde el primer nivel corresponde con "totalmente en desacuerdo" y el quinto nivel "muy de acuerdo".

3. Adaptación del Inventario de Funciones del Voluntariado al Voluntariado Español, Dávila y Chacón (2003): Este cuestionario mide las causas de la motivación en el voluntariado. Consta de 30 ítems. Para responderlos se utiliza una escala de siete puntos que va de 1 (no importante) a 7 (extremadamente importante). En él se puede encontrar los siguientes aspectos: defensa del yo, valores, mejora del currículum, relaciones sociales, conocimiento y mejora del estado de ánimo.

\section{Procedimiento}

A los participantes se les administraron los cuestionarios en su lugar de trabajo, les informamos del propósito de nuestro estudio y de la confidencialidad de la información que nos prestaban. A los voluntarios se les aplicaron los tres cuestionarios citados anteriormente ya los trabajadores remunerados el Cuestionario de Compromiso Q12 y el Cuestionario de Satisfacción Laboral S21/26. El tiempo medio que empleaban los participantes en contestar los cuestionarios fue de 10 minutos en el caso de los trabajadores remunerados y 15 en el caso de los trabajadores voluntarios.

\section{Resultados}

En primer lugar, para comprobar las diferencias significativas de los diferentes aspectos enmarcados dentro del cuestionario de satisfacción S26/21, se realizó una prueba de $\mathrm{t}$ de student para muestras independientes. Asumiendo $p<.05$, los resultados mostraron diferencias entre ambos grupos en todas las categorías. En cuando a la satisfacción en la supervisión y participación en la organización se presenta una $\mathrm{t}(21.56)=-3.63, p=.001$, la satisfacción con la remuneración y las prestaciones con $\mathrm{t}(27.83)=-2.47, p=.02$, la satisfacción intrínseca con $\mathrm{t}(14.00)=-5.07$, $p<.001$, la satisfacción con el ambiente físico con $t(24.07)=-2.168, p=.04$, la satisfacción con la cantidad de producción con $\mathrm{t}(19.24)=-3.55, p=.001$ y la satisfacción relacionada con la calidad con $\mathrm{t}(22.14)=-3.18, p=.004$.

Gráfica 1. Medias de satisfacción en los grupos "trabajadores" y "voluntarios"

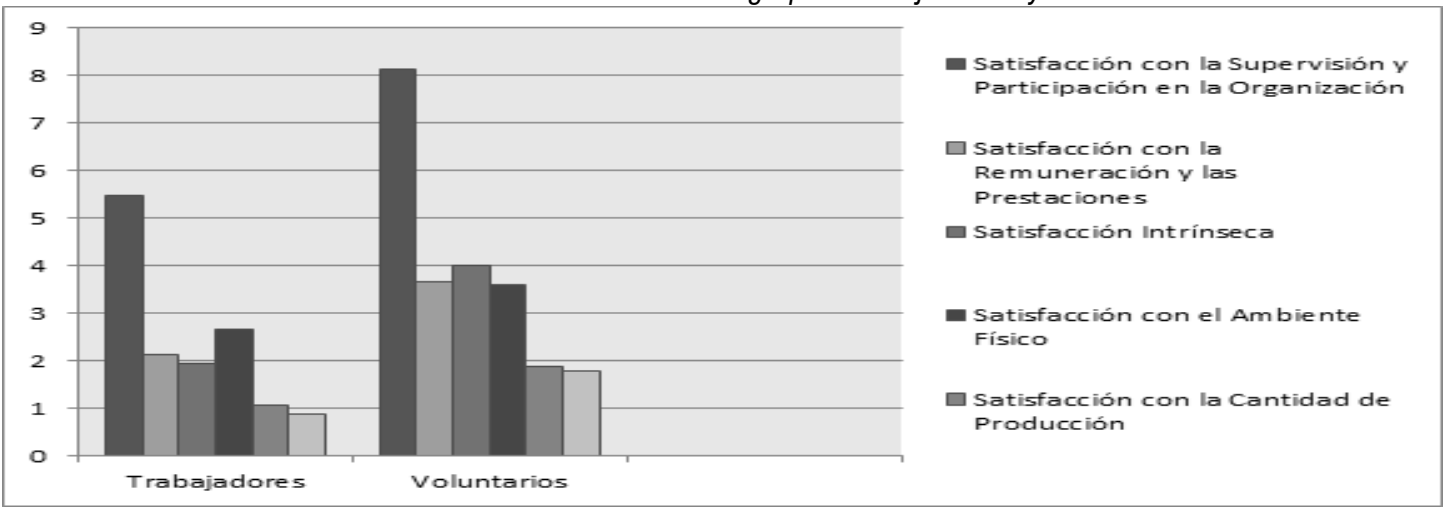


En cuanto al cuestionario de satisfacción laboral S21/26, encontramos que la satisfacción laboral es significativamente mayor en todas las categorías en el grupo de voluntariado frente al de trabajadores remunerados. Las que más diferencias muestran son la correspondiente a la satisfacción con la supervisión y participación de la organización, donde la media de los trabajadores es de 5.47 (DT=2.503) frente a 8.13 (DT=1.356) de voluntarios, y la satisfacción intrínseca, con unos valores de 1.93 $(D T=1.580)$ en trabajadores en contraposición a $4(D T=0)$ en voluntarios.

En categorías como la satisfacción con el ambiente, la cantidad y la calidad los voluntarios tienen resultados como 3.60 (DT=0.910); $1.87 \quad(\mathrm{DT}=0.352) ; 1.80$ (DT=0.561) frente a 2.67 (DT=1.397); 1.07 (DT=0.799) y 0.87 (DT=0.990) en trabajadores. La satisfacción con las prestaciones también es mayor en voluntarios con un 3.67 (DT=1.633) mientras que la media de los trabajadores es de 2.13 (DT=1.767). Las desviaciones típicas, nos demuestran que las respuestas correspondientes al grupo de trabajadores tienen una mayor variabilidad en la tendencia de respuestas en comparación con la del grupo voluntariado.

Seguidamente, para comprobar si existen diferencias en el compromiso se realizó otra prueba $\mathrm{t}$ de student donde $\mathrm{t}(21.11)=-4.57, \quad p<.001$, definiéndose diferencias significativas.

Gráfica 2. Diferenciación de medias en el compromiso en los grupos "trabajadores" y "voluntarios"

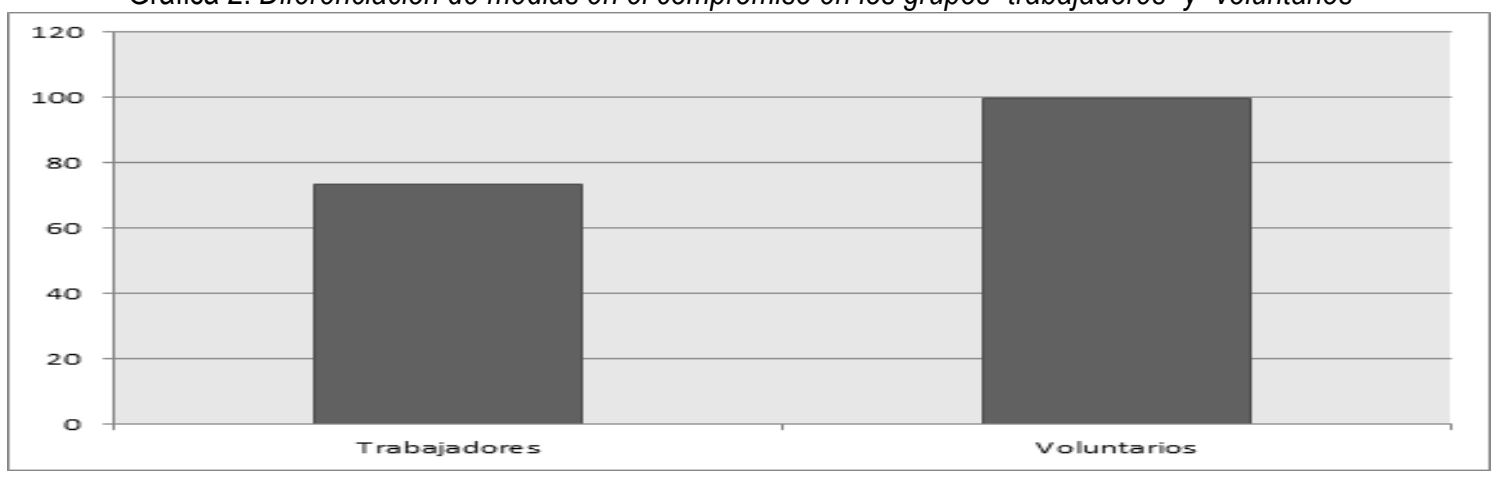

En lo que se refiere al Cuestionario de compromiso Q12 la diferencia de medias total existentes entre el 73.47 (DT=19.584) en trabajadores remunerados y el 99.53 $(\mathrm{DT}=10.225)$ en voluntarios, demuestra la diferencia significativa en torno a las diferencias de grupo.

Gráfica 3. Medias de motivación para el grupo "voluntarios"

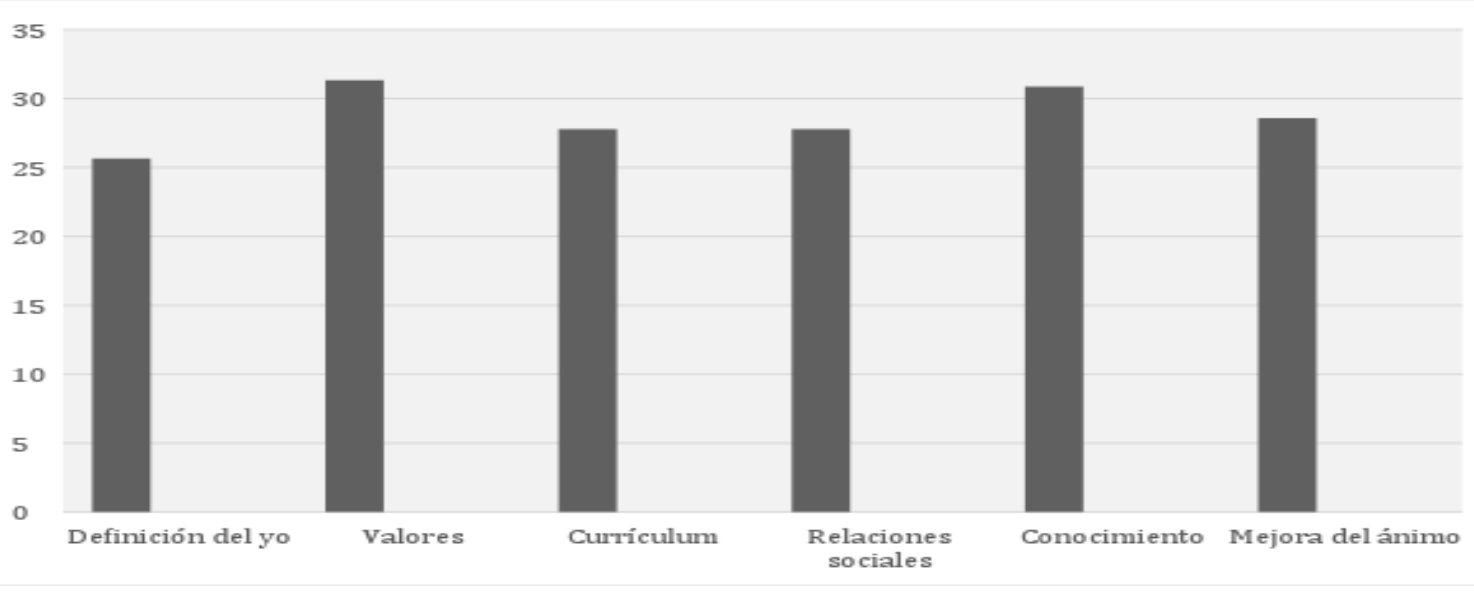


Los resultados obtenidos en el Cuestionario de Adaptación del Inventario de Funciones del Voluntariado se observan que todos los valores se mantienen dentro de unos rangos comprendidos entre 25 y 32 . El menos observable y en el que se encuentran las puntuaciones más bajas es la definición del yo $(M=25.67)$, seguido de otros como la mejora de currículum $(M=27.8)$ y las relaciones sociales $(M=27.8)$ y mejora del ánimo $(M=28.6)$. Sin embargo, las categorías que mayor puntuación han recibido fueron la correspondiente a la adquisición de valores $(M=31.33)$ y de conocimientos $(M=38.87)$.

\section{Discusión}

Una vez aplicadas las herramientas mencionadas a la muestra, podemos confirmar la hipótesis propuesta de que los voluntarios trabajan con una mayor satisfacción y compromiso que los empleados remunerados, lo que también se ha relacionado con un alto nivel de motivación en aquellos colectivos que trabajan de manera desinteresada (voluntarios). Ello quiere decir que las diferencias encontradas han sido significativas desde el punto de vista estadístico en las tres variables dependientes (compromiso, satisfacción y motivación -voluntarios-).

Además, se puede añadir que, en el grupo de las personas altruistas, los aspectos más destacados de la motivación han sido de carácter intrínseco. Las motivaciones que mueven a los voluntarios en la mayoría de los casos son los valores, el afán de conocimiento y la mejoría que conlleva dicha actividad en su estado de ánimo.

Se ha considerado que un correcto estudio del compromiso, satisfacción y motivación que mueve a los grupos humanos a trabajar puede tener aplicaciones muy variadas, no solo en el ámbito laboral, sino también en el ámbito académico y social. Extrapolar las conclusiones extraídas de estos estudios (que van en la misma línea) puede conllevar una mejoría significativa no solo en las relaciones laborales, sino en sus resultados y la eficacia de estos, con un mayor conocimiento de los aspectos que se deben reforzar para que esto ocurra. Con respecto al estudio de Valor-Segura (2011), se han encontrado la idea de múltiple causalidad de las actitudes en el voluntariado, respaldándose la idea de que se mueven por una cuestión de valores y bienestar propio $y$, no encontrándose motivaciones de pertenencia a un grupo, sino de ampliación del conocimiento (Valor-Segura, 2011). Aun así, si se ha encontrado que las relaciones sociales son importantes para este colectivo. Estos datos también respaldan la Teoría Funcional aplicada al voluntariado que propone una causalidad multifactorial del comportamiento de los voluntarios (Chacón y col., 2007; Clary y Snyder, 1991 y 1999; López, Cábanas y Chacón, 1997; Omoto y Snyder, 1995). Así mismo, podría confirmarse la propuesta de Bandura en su Teoría del Aprendizaje Social (1977) de que la experiencia vivida es la que modula el comportamiento desinteresado, no dándose motivaciones extrínsecas en sujetos con buenas experiencias dentro de este ámbito y han interiorizado estos beneficios.

\section{Referencias}

Arciniega, L. (2002). Compromiso Organizacional en México: ¿Cómo hacer que la gente se ponga la camiseta? Dirección Estratégica, Revista de Negocios del ITAM, 2(1), 21-23.

Betanzos Díaz, N., Paz Rodríguez, F. (2007). Análisis psicométrico del compromiso organizacional como variable actitudinal. Anales de Psicología, 23(2), 207-215.
Bandura, A. (1977). Social learning theory. Englewood Cliffs, NJ: Prentice-Hall.

Brief, AP., \& Weiss, HM. (2001). The affective dimensions of organizational behavior, Working paper, Tulane University.

Cábanas, M. y Chacón, F. (1997). Intervención psicosocial y servicios sociales. Madrid: Síntesis. 
Chacón, F., Pérez, T., Flores, J. y Vecina, ML. (2010). Motivos del voluntariado: categorización de las motivaciones de los voluntariados mediante pregunta abierta. Intervención psicosocial: Revista sobre igualdad y calidad de vida, 19, 213-222.

Chiang MM. y Ojeda JF (2013). Estudio de la relación entre satisfacción laboral y el desempeño de los trabajadores de las ferias libres. Contaduría y Administración, 58 (2), 39-60.

Clary, EC., Snyder, M., Ridge, RD., Copeland, J., Stukas, AA., Hauger, J., \& Miene, P. (1998). Understanding and assessing the motivations of volunteers: a functional approach. Journal of Personality and Social Psychology, 74, 1516-1530.

Gallardo, E. y Triadó, X. (2007). Revisión de las aportaciones teóricas sobre el compromiso organizativo: ¿acaso importan las actitudes? XXI Congreso anual de la Academia Europea de Dirección y Economía de la Empresa, Universidad Rey Juan Carlos - Madrid.

Mañas Rodríguez, M., Salvador, C., Boada, J., González, E. y Agulló, E. (2007). La satisfacción y el bienestar psicológico como antecedentes del compromiso organizacional. Psicothema, 19(3), 395-400.

McDonald, DJ., \& Makin, PJ. (2000) The Psychological Contract, Organizational Commitment and Job Satisfaction of Temporary Staff. Leadership \&amp; Organization Development Journal, 21, 84-91.

Omoto, AM., \& Snyder, M. (1995). Sustained helping without obligation: Motivation, longevity of service, and perceived attitude change among AIDS volunteers. Journal of Personality and Social Psychology, 68(4), 671-686.

Robbins, S. (2004). Comportamiento organizacional. México: Pearson Educación.

Salessi, S. y Omar, A. (2017). Satisfacción laboral: un modelo explicativo basado en variables disposicionales. Revista Colombiana de Psicología, 26(2), 329-345.

Valor-Segura, I. y Rodríguez-Bailón, R. (2011). Motivaciones, compromiso y experiencia voluntaria en estudiantes universitarios españoles. Anales de Psicología, 27, 157-164.

Vecina, ML. y Chacón, F. (2013). ¿Es el engagement diferente de la satisfacción y del compromiso organizacional? Relaciones con la intención de permanencia, el bienestar psicológico y la salud física percibida en voluntarios. Anales de Psicología, 29, 225-232. 